\title{
Following Your Heart or Your Head: Focusing on Emotions Versus Information Differentially Influences the Decisions of Younger and Older Adults
}

\author{
Joseph A. Mikels, Corinna E. Löckenhoff, \\ Sam J. Maglio, and Laura L. Carstensen \\ Stanford University
}

\author{
Mary K. Goldstein and Alan Garber \\ VA Palo Alto Health Care System, Palo Alto, CA, and \\ Stanford University
}

\begin{abstract}
Research on aging has indicated that whereas deliberative cognitive processes decline with age, emotional processes are relatively spared. To examine the implications of these divergent trajectories in the context of health care choices, we investigated whether instructional manipulations emphasizing a focus on feelings or details would have differential effects on decision quality among younger and older adults. We presented 60 younger and 60 older adults with health care choices that required them to hold in mind and consider multiple pieces of information. Instructional manipulations in the emotion-focus condition asked participants to focus on their emotional reactions to the options, report their feelings about the options, and then make a choice. In the information-focus condition, participants were instructed to focus on the specific attributes, report the details about the options, and then make a choice. In a control condition, no directives were given. Manipulation checks indicated that the instructions were successful in eliciting different modes of processing. Decision quality data indicate that younger adults performed better in the information-focus than in the control condition whereas older adults performed better in the emotion-focus and control conditions than in the information-focus condition. Findings support and extend extant theorizing on aging and decision making as well as suggest that interventions to improve decision-making quality should take the age of the decision maker into account.
\end{abstract}

Keywords: aging, decision making, emotion, affect heuristic, working memory

Health care decisions in the United States are characterized by an increasing emphasis on patient autonomy (Botti \& Iyengar, 2006). At the same time, medical choices have become increasingly complex and pose a considerable challenge to many people (e.g., Hibbard, Slovic, Peters, Finucane, \& Tusler, 2001). As a

Joseph A. Mikels, Corinna E. Löckenhoff, Sam J. Maglio, and Laura L. Carstensen, Department of Psychology, Stanford University; Mary K. Goldstein and Alan Garber, VA Palo Alto Health Care System, Palo Alto, CA, and Center for Primary Care and Outcomes Research, Stanford University.

Joseph A. Mikels and Corinna E. Löckenhoff are now at Cornell University. Sam J. Maglio is now at New York University. This project was supported in part by NIA research Grant AG08816 to Laura L. Carstensen, NIA seed grant through the Center on Advancing Decision Making in Aging AG24957 to Joseph A. Mikels, Ruth L. Kirschstein National Research Service Award AG022264 to Joseph A. Mikels, and the NIA intramural research program to Corinna E. Löckenhoff. We thank Lauren Smith, Sarah Fairbank, Kaoru Nashiro, and Victoria Folayan for their invaluable assistance with data collection. We also thank Elaine Cheung for her incredible assistance with the preparation of this article. Finally, we thank an anonymous reviewer for helpful suggestions in developing the theoretical and practical implications of the current study. Views expressed are those of the authors and not necessarily those of the Department of Veterans Affairs.

Correspondence concerning this article should be addressed to Joseph A. Mikels, Department of Human Development, Cornell University, Martha Van Rensselaer Hall, Ithaca, NY 14853-4401. E-mail: jmikels@ cornell.edu result, the ways in which people make decisions about health care constitute an important target of research for behavioral interventions. Moreover, research on aging suggests that no single type of intervention will suit all age groups (see, e.g., Carstensen, Mikels, \& Mather, 2006; Löckenhoff \& Carstensen, 2004; Peters, Hess, Vastfjall, \& Auman, 2007). The present study extends research on age differences in information processing to the health domain by implementing instructional manipulations that emphasize either affective or deliberative approaches to decision making and by examining their effect on decision quality among younger and older adults.

Traditionally, psychologists and economists have presumed (if tacitly) that good decisions are the product of analytic and deliberative processing. Yet, recent dual process theories of decision making postulate that affective/experiential and deliberative processes may operate in parallel and may be comparably influential (Kahneman, 2003; Loewenstein, Weber, Hsee, \& Welch, 2001; Reyna, 2004; Slovic, Peters, Finucane, \& MacGregor, 2005). In fact, Slovic et al. (2005) introduced the concept of the "affect heuristic" and suggested that "using an overall, readily available affective impression can be easier and more efficient than weighing the pros and cons of various reasons or retrieving relevant examples from memory" (p. S36). Such considerations are especially relevant across the adult life span as research suggested that affective and deliberative processing abilities show differential age trajectories (Carstensen et al., 2006; Peters et al., 2007). More important, the above description of the affect heuristic draws a concrete and specific distinction between two dual processes: 
affect versus memory. This specific contrast between feelings and memory for details allows for a precise distinction that can be operationally defined. Drawing on these considerations, reliance on processes that encode affective impressions versus the details into memory would allow for a precise test of the efficacy of these dual processes. As such, the central goal of the current project was to examine the differential outcomes of affect- versus detailfocused encoding on decision-making performance in the health domain. Such considerations are especially relevant for understanding health care choices across the life span as research suggests that affective and deliberative processing abilities show differential age trajectories (Carstensen et al., 2006; Peters et al., 2007).

Fluid intelligence, that is, deliberative/effortful processing, peaks early in life followed by a steady decline thereafter (for reviews, see Craik \& Salthouse, 2008). This component of intelligence comprises several subcomponents that all show consistent age-related decline including speed of information processing, temporary storage of information (i.e., short-term memory), and the storage and manipulation of information (i.e., working memory, for a review, see Baltes, 1997). Emotional processing, in contrast, appears to be well maintained at older ages (for a review, see Carstensen et al., 2006). More important, this selective preservation of emotional processing is found even in working memory (Mikels, Larkin, Reuter-Lorenz, \& Carstensen, 2005). Specifically, whereas age is negatively associated with working memory performance in virtually all content domains (see, e.g., Babcock \& Salthouse, 1990; Verhaeghen, Marcoen, \& Goossens, 1993), Mikels et al. (2005) found that working memory for emotional information was selectively unimpaired. Insofar as working memory is the central cognitive system involved in the maintenance and manipulation of information (Baddeley, 1986; Baddeley \& Hitch, 1974), age-related changes in working memory have significant implications for the decision context because increasingly complex decisions place high demands on processing capacity. Indeed, age-related declines in two measures of fluid intelligence, shortterm memory and processing speed, have been linked to decreased decision-making competence (see, e.g., Finucane, Mertz, Slovic, \& Schmidt, 2005). Thus, decision tasks that place high load on fluid intelligence may result in decisions of lower quality for older individuals.

In keeping with the old adage that one should always "read the small print" before making a choice, current policies aimed at improving medical decisions tend to emphasize the concept of "informed choice" and encourage in-depth processing of all available information (see Hibbard et al., 2001). Although this approach may be successful in motivating younger adults to make the best use of their fluid cognitive capacity (such as working memory for verbal information), it does little to address age-related decrements in effortful processing abilities. In light of age-related declines in deliberative cognitive abilities, it is perhaps not surprising that older adults make decisions differently than their younger counterparts (Sanfey \& Hastie, 2000). For instance, older adults appear to be less flexible in revising judgment and choice strategies (Chasseigne, Mullet, \& Stewart, 1997), prefer cognitive strategies that are less demanding (Johnson, 1990, 1993), and are relatively hesitant to make decisions (Dror, Katona, \& Mungur, 1998). Moreover, with respect to aging and health care decisions, evidence suggests that older adults seek less information, are less systematic, and make decisions more quickly relative to younger adults (Löckenhoff \& Carstensen, 2004; Meyer, Russo, \& Talbot, 1995). Although such strategies do not necessarily lead to worse decision outcomes (Meyer et al., 1995), the observed age effects indicate that age groups differ in their approach to complex decisions and suggest that older adults may find such decisions more difficult than younger adults (e.g., Finucane et al., 2005, 2002). In particular, older adults display deficits in comprehension of relevant information when making decisions. Given these age-related changes in deliberative decision processes, older adults may be better served by leveraging their comparatively well-preserved emotional processing capacity to help offset difficulties arising from age-related declines.

To examine this possibility, the present study examined the relative influence of age and decision strategy on decision quality in the context of health care choices. We chose to focus on health care rather than other important consumer domains due to its timeliness, universal salience, and the richness and complexity of information available to consumers of health care. The design of our paradigm was based on previous research on working memory (e.g., Daneman \& Carpenter, 1980; Mikels et al., 2005) and required participants to hold multiple pieces of information in mind and weigh them against each other. Thus, our design is an empirically grounded and theoretically consistent approach that was applied to the domain of health care decision making. To target working memory processes within a complex decision task, we integrated a standard procedure used in working memory research, namely, increasing the number of attributes presented for each choice across trials. To examine the role of decision strategy, instructional manipulations directed participants' attention either to the details of the available information (information-focus condition) or to their overall emotional reactions to the choice alternatives (emotion-focus condition). In a control condition, no directives were given. We predicted that older adults would perform better in the emotion-focus condition than in the information-focus condition, whereas younger adults would show the opposite pattern.

\section{Method}

\section{Participants}

Sixty younger adults aged 18 to 30 years and 60 older adults aged 65 to 85 years were recruited from the San Francisco Bay area by a survey research firm and paid for their participation. Participants were screened for dementia as well as neurological and affective disorders. Three participants were replaced as a result of these screenings or computer malfunction. Table 1 describes the two age groups in detail. They did not differ by ethnicity, education, scaled income, or self-reported health. More important, within each age group, there were no significant differences in any of these participant characteristics as a function of condition.

\section{Materials}

We constructed a series of hypothetical choices among pairs of health care plans, physicians, medical treatments, and homecare aides. Fictitious names (e.g., "Care Net," "Betatine") were generated for health care plans and treatment options. Names of physi- 
Table 1

Participant Characteristics by Age Group

\begin{tabular}{|c|c|c|c|c|c|c|}
\hline \multirow[b]{2}{*}{ Characteristic } & \multicolumn{2}{|c|}{ Younger $N=60$} & \multicolumn{2}{|c|}{ Older $N=60$} & \multicolumn{2}{|c|}{ Statistic } \\
\hline & $M$ & $S D$ & $M$ & $S D$ & $t(118)$ & $p$ \\
\hline Age (in years) & 22.58 & 3.00 & 72.43 & 5.11 & & \\
\hline \multicolumn{7}{|l|}{ Sex } \\
\hline Female & $48 \%$ & & $48 \%$ & & & \\
\hline Male & $52 \%$ & & $52 \%$ & & & \\
\hline \multicolumn{7}{|l|}{ Ethnicity } \\
\hline African American & $35 \%$ & & $35 \%$ & & & \\
\hline European American & $65 \%$ & & $65 \%$ & & & \\
\hline Education (in years) & 14.59 & 1.51 & 15.19 & 2.35 & 1.65 & $>.1$ \\
\hline Scaled income & 2.93 & 0.98 & 2.77 & 1.00 & .92 & $>.3$ \\
\hline Self-reported health (Wahler) & 42.25 & 25.46 & 39.42 & 20.78 & 0.67 & $>.5$ \\
\hline Vocabulary (WAIS-III) & 45.15 & 11.35 & 44.52 & 12.31 & 0.29 & $>.7$ \\
\hline Digit-symbol coding (WAIS-III) & 85.92 & 16.24 & 55.62 & 11.95 & 11.64 & $<.001$ \\
\hline Digit span (WAIS-III) & 19.05 & 3.62 & 17.07 & 3.97 & 2.86 & $<.01$ \\
\hline
\end{tabular}

Note. Scaled income was based on a scale ranging from 1 (lower income) to 5 (upper income); self-reported health (Wahler, 1973) was based on a rating of 42 different symptoms on a scale ranging from 0 to 5 , maximum score $=210$; Vocabulary from the Wechsler Adult Intelligence Scale (WAIS-III; Wechsler, 1997): maximum score $=66$; digit-symbol coding from the WAIS-III: maximum score $=133$; digit span from the WAIS-III: maximum score $=30$.

cians and homecare aides were selected from the 1990 census index of frequent last names (e.g., "Dr. Smith," "Aide Young"). Individual choice attributes describing each option (e.g., "you cannot obtain health care outside of your network") were adapted from a prior study (Löckenhoff \& Carstensen, 2007). ${ }^{1}$

For each decision, participants chose between two different options described by a series of attributes presented sequentially. Given our interest in placing high load on the working memory component of the decision process, we based the design of our decision task on Daneman and Carpenter's (1980) reading span task. Similar to their task, working memory load increased across trials from one to four attributes per option (corresponding to memory loads of two, four, six, or eight). At each load level, participants made one choice in each of the four domains for a total of 16 trials.

To assess choice quality, each decision was constructed such that one option had a higher proportion of positive to negative attributes. More important, beyond this a priori approach to constructing the "best" option, we also collected importance ratings from each participant with which to weigh the options for an alternative scoring procedure (described in greater detail below). The order of the best option was counterbalanced across participants. Also, each individual attribute was phrased positively (e.g., "dental care is fully covered") for half of the participants and negatively (e.g., "dental care is not covered") for the other half. The assignment of individual attributes to load levels was randomized across participants. See the Appendix for sample trial attributes at a load of eight.

\section{Additional Measures (After Decision Trials)}

To assess general cognitive functioning, participants completed several measures from the Wechsler Adult Intelligence Scale (WAIS-III; Wechsler, 1997) including processing speed (digit symbol), short-term memory (digit span), and knowledge (vocabulary). They also completed a measure of self-reported health
(Wahler, 1973). As a manipulation check, participants reported on a 7-point scale ranging from 1 (not at all) to 7 (extremely) the extent to which they focused on the specific details and separately the extent to which they focused on their feelings when making the decisions. Participants also reported the extent to which making the decisions made them feel positive and negative emotions on a 7-point scale ranging from 1 (not at all) to 7 (extremely). To assess previous experience with similar choices, participants indicated the number of choices they had previously made in each of the decision domains. Finally, participants rated the personal importance of each individual choice attribute (e.g., "whether dental care is covered") on a 7-point scale ranging from 1 (not at all important) to 7 (very important). The items presented for these importance ratings were not positively or negatively valenced, nor were they matched to the previously presented choice options.

\section{Procedure}

After providing written informed consent, participants were seated at a computer. Each participant was assigned to one of three conditions: control, information focus, or emotion focus. Instructional manipulations in the experimental conditions were adapted from previous studies (Kennedy, Mather, \& Carstensen, 2004; Löckenhoff \& Carstensen, 2007) and designed to elicit a focus on the specific details versus emotional reactions, respectively. Consistent with Slovic et al.'s (2005) description of the affect heuristic, emotion-focused instructions encouraged the formation of an overall affective impression of each choice option, whereas information-focused instructions were aimed at the retention of individual details about each option.

To ensure that the experimental instructions remained salient throughout the entire decision process, manipulations were in-

\footnotetext{
${ }^{1}$ Complete descriptions of the choice attributes are available from the authors.
} 
cluded before the choice trials, during the review of the information, and immediately after the information had been presented but before the choice was made.

It was first explained to all participants that they would be making a series of hypothetical health care decisions by choosing one of two options and that information regarding the decisions would be presented on the computer screen. Participants in the information-focus condition were instructed to attend to the specific details about each option, whereas participants in the emotion-focus condition were instructed to attend to their emotional reactions to the choice options. The control group received no instructions regarding focus.

After the initial instructions, participants completed two practice trials and then the 16 actual trials. Each trial began with a prompt indicating the decision domain (i.e., health care plans, physicians, medical treatments, and homecare aides). Then the attributes for each of two options were presented sequentially on the computer screen. To minimize confusion, the name of the choice option was repeated before each attribute defining that option, and the attributes for each choice option were presented in blocks.

To reinforce experimental manipulations during the choice trials, each piece of information was followed by a brief query. In the information-focus condition participants were asked to rate how well they remembered the details about a given choice option whereas in the emotion-focus condition they were asked to rate their feelings about the option. In the control condition they were simply asked to press the space bar to advance.

Finally, after the full set of attributes in each trial had been presented, an additional manipulation reinforced a focus on the details versus emotional reactions. Participants in the informationfocus condition were asked to write down as many details about each option as possible. Participants in the emotion-focus condition were asked to write down all of their feelings about each option. Participants in the control condition were asked to write down everything that came to mind about the options. Next, participants were asked to choose one of the options. Following the decision trials, participants completed the additional measures described above.

\section{Choice Quality Scoring Method}

As described above, choice trials were designed such that one option had a greater proportion of positive versus negative attributes than the other and was thus the dominating or "best" alternative. A choice was scored as "correct" when a participant chose the option with the greater proportion of positive attributes. To assess "choice quality," we computed the percentage of correct choices across trials within each level of cognitive load. Given the design of the current study, determining choice quality with a "tally" of positive versus negative attributes for each trial seemed to be the most parsimonious scoring method. However, it is possible that the participants used a "weighted additive strategy," taking into account the positive or negative value and the subjective relevance of the attributes. To examine this alternative, we also computed choice quality as a weighted score (based on the individual importance ratings gathered at the end of the experiment). Critically, the pattern of results did not differ when weighted correct responses were used as the dependent variable. Here we report the tally results.

\section{Results}

\section{Descriptive Analyses}

As can be seen in Table 1, older adults performed more poorly than younger adults on measures from the WAIS-III (Wechsler, 1997) assessing processing speed (digit symbol) and short-term memory (digit span), but did not differ from younger adults on a measure of knowledge (vocabulary). More important, within each age group, there were no significant differences in any of these cognitive measures as a function of condition. Finally, as measured by the number of specific experiences participants had with previous choices in each decision domain, older adults reported greater experience $(M=3.47, S D=4.65)$ relative to younger adults $(M=1.59, S D=0.72), t(118)=3.09, p<.005$.

\section{Manipulation Check Analyses}

As described above, questions were included to assess the extent to which participants focused on the details versus their feelings while making the decisions. Responses to these two questions were analyzed using separate analyses of variances (ANOVAs) with age (young, old) and condition (control, emotion focus, information focus) as between-subjects variables. These data are presented in Table 2. With respect to detail-focus, analyses revealed a main effect of age, in that older adults overall reported less focus on details relative to younger adults, $F(1,114)=3.94, p<.05, \eta_{\mathrm{p}}^{2}=$ .03. The main effect of condition and the age by condition interaction were not significant. With respect to feeling focus, the analyses only revealed a condition main effect, $F(1,114)=8.54$, $p<.001, \eta_{\mathrm{p}}^{2}=.13$ (see Table 2). Specifically, although the emotion-focus and control conditions did not differ in self-reported feeling focus, $t(78)=1.73, n s$, both the emotion-focus and control conditions did report a greater focus on feelings relative to the information-focus condition, $t(78)=4.11, p<.001$, and $t(78)=$ $2.36, p<.05$, respectively. These findings suggest that our experimental manipulations were indeed effective in that participants in the emotion-focus condition relied to a greater extent on their feelings than those in the information-focus condition. In addition, these findings suggest that participants in the control condition also relied quite a bit on their feelings.

Second, as part of our experimental manipulation before making each choice, we had participants in the information-focus condition report the details about each option, in the emotion-focus condition report their feelings about each option, and in the control condition report everything that came to mind. Although these manipulations represent different requests, we were able to code the number of total attributes referenced in these reports and examined if the percentage of attributes referenced differed between the conditions and age groups. These data were analyzed using an ANOVA with age (young, old) and condition (control, emotion focus, information focus) as between-subjects variables. These data are presented in Table 2. This analysis only revealed main effects of condition and age group. In particular, the age group main effect indicates that across all conditions, older adults referenced fewer attributes than the younger adults, $F(1,114)=$ 29.40, $p<.001, \eta_{\mathrm{p}}^{2}=.21$. More important, though, the condition main effect indicates that the participants in the emotion-focus condition referenced fewer attributes than both the information- 
Table 2

Manipulation Check Data Broken Down by Instructional Condition and Age Group

\begin{tabular}{lcccc}
\hline \multicolumn{1}{c}{ Variable } & \multicolumn{4}{c}{ Conditions } \\
\cline { 2 - 4 } & Control & Emotion focus & Information focus & $M$ \\
\hline $\begin{array}{l}\text { Self-reported detail focus } \\
\quad \text { Young }\end{array}$ & $5.7(1.2)$ & $5.6(1.2)$ & $5.6(1.2)$ & $5.6(1.2)$ \\
$\quad$ Old & $5.6(1.3)$ & $5.1(1.3)$ & $4.8(1.3)$ & $5.2(1.3)$ \\
$\quad M$ & $5.7(1.2)$ & $5.3(1.3)$ & $5.2(1.3)$ & $5.4(1.3)$ \\
Self-reported feeling focus & & & & \\
$\quad$ Young & $5.0(1.3)$ & $5.6(1.1)$ & $4.2(1.8)$ & $4.9(1.5)$ \\
$\quad$ Old & $4.8(1.7)$ & $5.3(1.2)$ & $3.9(2.0)$ & $4.7(1.7)$ \\
$\quad M$ & $4.9(1.5)$ & $5.4(1.2)$ & $4.0(1.9)$ & $4.8(1.6)$ \\
Percentage attributes referenced & & & & \\
$\quad$ Young & $61.8(19.3)$ & $41.5(17.4)$ & $70.3(12.6)$ & $57.8(20.4)$ \\
$\quad$ Old & $46.8(18.6)$ & $26.1(18.1)$ & $48.9(17.6)$ & $40.6(20.6)$ \\
$M$ & $54.3(20.2)$ & $33.8(19.2)$ & $59.6(18.5)$ & $49.2(22.2)$ \\
\hline
\end{tabular}

Note. Standard deviations are shown in parentheses.

focus and control conditions, $F(2,114)=24.54, p<.001, \eta_{\mathrm{p}}^{2}=$ .30. Thus, these analyses also suggest that our experimental manipulations were effective in that participants in the informationfocus condition referred to more attributes relative to the emotionfocus condition. It is notable that participants in the control condition considered as many attributes as those participants in the information-focus condition.

\section{Main Analyses}

To test our main hypotheses, we computed a repeated-measures ANOVA with age (young, old) and condition (control, emotionfocus, information-focus) as between-subjects variables, working memory load $(2,4,6,8)$ as a within-subject variable, and choice quality (i.e., the percentage of correct choices) as the dependent variable. The means broken down by all of these factors are presented in Table 3. Mauchly's $W$ was $.97, n s$, indicating that sphericity could be assumed. There was a main effect of load, $F(3$, $112)=19.31, p<.001, \eta_{\mathrm{p}}^{2}=.34$, and within-subject contrasts revealed a linear decline in choice quality with increasing load, $F(1,114)=49.48, p<.001, \eta_{\mathrm{p}}^{2}=.30$ (see Table 3 ). Thus, increasing the number of attributes placed significant load on working memory making the task reasonably difficult, especially at higher loads. A main effect of age group, $F(1,114)=8.16, p<$ $.01, \eta_{\mathrm{p}}^{2}=.046$, indicated that across loads and conditions participants in the younger group $(M=81.0 \%, S E=1.3)$ outperformed participants in the older group $(M=75.8 \%, S E=1.3)$. The main effect of condition was not significant, but there was an age group by condition interaction, $F(2,114)=6.83, p<.005, \eta_{p}^{2}=.11$ (see Figure 1). For the younger adults, choice quality was higher in the information-focus condition relative to the control condition, $t(38)=2.08, p<.05$, whereas choice quality in the emotion-focus condition did not differ from choice quality in the control or

Table 3

Mean Choice Quality by Instructional Condition, Age Group, and Load

\begin{tabular}{lccccc}
\hline & \multicolumn{5}{c}{ Cognitive Load } \\
\cline { 2 - 6 } \multicolumn{1}{c}{ Variable } & 2 & 4 & 6 & 8 & $M$ \\
\hline $\begin{array}{l}\text { Information focus } \\
\quad \text { Young }\end{array}$ & $92.5(14.3)$ & $85.0(20.5)$ & $80.0(17.4)$ & $80.0(20.8)$ & $84.4(8.2)$ \\
$\quad$ Old & $84.6(22.3)$ & $68.3(15.9)$ & $68.3(17.9)$ & $59.2(26.8)$ & $70.1(11.2)$ \\
$\quad M$ & $88.5(18.9)$ & $76.7(20.0)$ & $74.2(18.4)$ & $69.6(25.9)$ & $77.2(12.1)$ \\
Emotion focus & & & & & \\
$\quad$ Young & $92.5(11.8)$ & $85.0(17.0)$ & $82.5(16.4)$ & $63.7(20.6)$ & $80.9(8.5)$ \\
$\quad$ Old & $87.5(15.2)$ & $78.8(16.8)$ & $75.4(25.4)$ & $69.2(24.8)$ & $77.7(10.5)$ \\
$\quad M$ & $90.0(13.6)$ & $81.9(17.0)$ & $79.0(21.4)$ & $66.5(22.7)$ & $79.3(9.6)$ \\
Control & & & & & \\
$\quad$ Young & $86.2(20.6)$ & $75.0(22.9)$ & $75.0(19.9)$ & $75.0(14.0)$ & $77.8(11.9)$ \\
$\quad$ Old & $92.5(14.3)$ & $75.0(21.5)$ & $76.2(22.2)$ & $75.0(22.9)$ & $79.7(9.0)$ \\
$\quad M$ & $89.4(17.8)$ & $75.0(21.9)$ & $75.6(20.8)$ & $75.0(18.8)$ & $78.8(10.5)$ \\
Total & & & & & \\
$\quad$ Young & $90.4(16.0)$ & $81.7(20.5)$ & $79.2(17.9)$ & $72.9(19.7)$ & $81.0(10.0)$ \\
$\quad$ Old & $88.2(17.6)$ & $74.0(18.4)$ & $73.3(22.0)$ & $67.8(25.3)$ & $75.8(10.9)$ \\
$M$ & $89.3(16.8)$ & $77.9(19.8)$ & $76.3(20.2)$ & $70.4(22.7)$ & $78.4(10.7)$ \\
\hline$\quad$
\end{tabular}

Note. Standard deviations are shown in parentheses. 


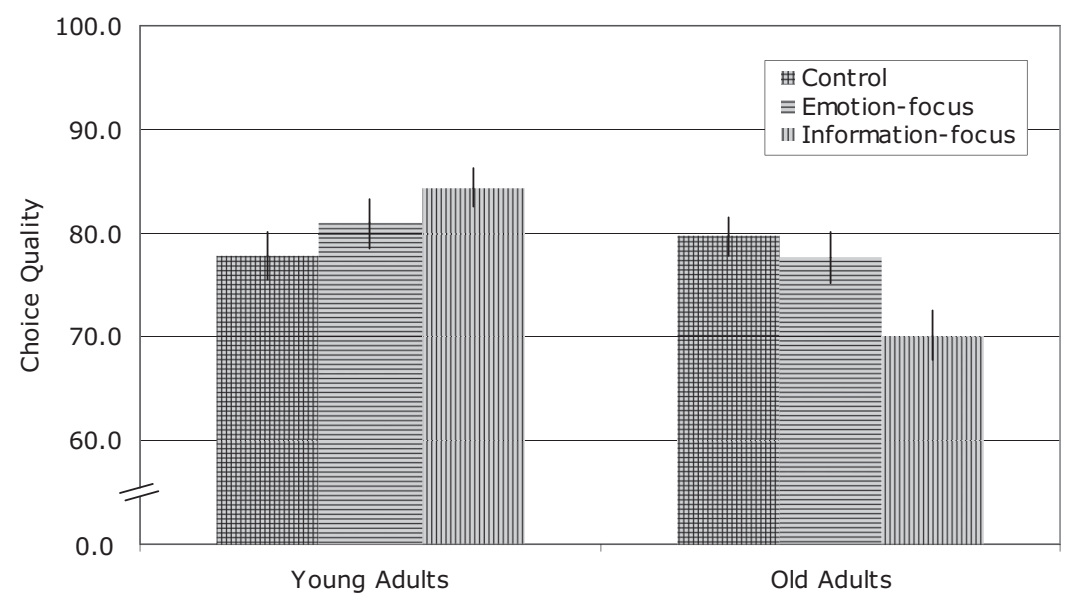

Figure 1. Mean choice quality by age group and condition. Bars are labeled with the corresponding means. Error bars show standard errors.

information-focus conditions, $t(38)=1.11, p>.25$, and $t(38)=$ $1.30, p>.20$, respectively. In contrast, older adults' choice quality was higher in the emotion-focus and control conditions relative to the information-focus condition, $t(38)=2.21, p<.05$, and $t(38)=3.05, p<.005$, respectively. When comparing age groups within each condition, choice quality in the information-focus condition was lower for older than for younger adults, $t(38)=$ $4.52, p<.001$, whereas in the emotion-focus and control conditions, older adults' choice quality was commensurate with younger adults' performance, $t(38)=0.84, p>.35$, and $t(38)=0.86, p>$ .39 , respectively. No other interactions reached significance.

To examine whether choice quality was related to cognitive ability, experience, decision-related emotions, and self-reported health status, we conducted a series of additional analyses. First, we correlated mean choice quality (collapsed across loads) with the measures of cognitive functioning, reports of experience, decision-related emotions, and self-reported health within each condition and age group. In the information-focus condition, the correlations with digit symbol reached significance for both younger and older adults, $r(20)=.45, p<.05$, and $r(20)=.45$, $p<.05$, respectively. None of the other correlations were significant. Moreover, conducting analyses of covariances (ANCOVAs) with digit symbol scores as the covariate, the age difference in the information-focus condition was no longer significant, $F(1,39)=$ $2.40, p>.12$, whereas results in the other conditions remained unchanged.

Finally, we examined age and condition effects on decisionrelated emotions using a repeated-measures ANOVA with age (young, old) and condition (control, emotion focus, information focus) as between-subjects factors and positive and negative decision-related emotions as a within-subject factor. See Table 4 for the means from this analysis. This analysis only revealed a condition by valence interaction, $F(2,114)=3.17, p<.05, \eta_{\mathrm{p}}^{2}=$ .05 . The simple effects indicate that participants in the emotionfocus condition reported a higher level of positive emotions relative to negative emotions when making the decisions, $t(39)=3.21$, $p<.005$, whereas there were no differences in positive and negative emotions in the other conditions.

\section{Discussion}

Based on previous research suggesting age-related declines in deliberative processing but relatively preserved emotional processing, we postulated that choice quality in older and younger adults would differ across conditions that encouraged a reliance on working memory for information versus emotion. Specifically, we expected that older adults would benefit more from instructions encouraging a focus on emotion while younger adults would benefit more from instructions emphasizing information acquisition.

The results are generally consistent with this hypothesis. Younger adults performed better in the information-focus condition than the control condition-suggesting that relative to their "default" approach (as observed in the control condition), younger adults make higher quality decisions when instructed to focus on deliberative processing. In contrast, older adults performed better in the emotion-focus and control conditions relative to the information-focus condition. Thus, although older adults performed better when focusing on emotional relative to deliberative processing, emotion-focus instructions did not elevate choice quality beyond the levels found in the control condition.

Table 4

Decision-Related Emotion Ratings by Instructional Condition and Age Group

\begin{tabular}{lccc}
\hline & \multicolumn{3}{c}{ Condition } \\
\cline { 2 - 4 } \multicolumn{1}{c}{ Variable } & Control & Emotion focus & Information focus \\
\hline Positive emotions & & & \\
$\quad$ Young & $4.3(1.5)$ & $4.4(1.2)$ & $3.9(1.4)$ \\
Old & $4.1(1.5)$ & $4.9(1.0)$ & $3.6(1.9)$ \\
$M$ & $4.2(1.5)$ & $4.6(1.1)$ & $3.8(1.7)$ \\
Negative emotions & & & \\
$\quad$ Young & $4.6(1.7)$ & $4.2(1.5)$ & $4.1(1.6)$ \\
Old & $4.0(1.8)$ & $3.4(1.5)$ & $3.8(1.6)$ \\
$M$ & $4.3(1.7)$ & $3.8(1.5)$ & $3.9(1.6)$ \\
\hline
\end{tabular}

Note. Standard deviations are shown in parentheses. 
Analyses of self-reported strategies and the number of referenced attributes provide some insight into the underlying processes. Consistent with instructions, participants in the emotionfocus condition reported greater reliance on feelings than participants in the information-focus condition. Further, although self-reported focus on details did not differ across conditions, participants in the information-focus condition referenced more attributes before making their choices compared to those in the emotion-focus condition. This is consistent with a more deliberative processing style. Taken together, these patterns suggest that the two experimental conditions successfully elicited different processing styles.

In the control condition, both older and younger adults appeared to rely on an amalgam of cognitive and emotional processes. Although the manipulation check data provide a glimpse of insight into the default processes employed by younger and older adults when no particular instructions are given, future research is needed to better elucidate the underlying processes. Across conditions, older adults reported less focus on details and referenced fewer attributes before making their choices. These patterns suggest that older adults generally rely less on detail-focused processing, but as observed in the control condition make decisions of considerably high quality, which is consistent with the proposition that older adults implement decision processes that optimize their performance (Mata, Schooler, \& Rieskamp, 2007). Younger adults, in contrast, performed better in the information-focus condition than in the control condition suggesting that younger adults can benefit from decision-enhancing interventions.

The present results have important theoretical and practical implications. From a theoretical point of view, socioemotional selectivity theory (SST; Carstensen, 2006) predicts that age-related changes in future time perspective shift motivational priorities from future-oriented information acquisition goals to presentoriented emotion-regulatory goals. Previous research has linked this age-related emphasis on emotion-regulatory goals to preferential processing of emotionally salient and positively valenced material among older relative to younger adults (Carstensen et al., 2006). The current results extend the postulates of SST by examining the consequences of differential processing styles on decision quality in the health care domain. Our findings also speak to Labouvie-Vief's (2003) dynamic integration of differentiation and optimization model, which suggests that different decision approaches may have different trade-offs across the life span. In particular, according to Labouvie-Vief, cognitive-affective complexity decreases from young adulthood into old age. As a result, younger adults may be better able to effectively employ a variety of strategies whereas older individuals may not. In support of this claim, we found that younger adults make decisions of high quality using both information-focus and emotion-focus strategies whereas older adults do not appear to be able to effectively employ an information-focused strategy.

The current findings also make theoretical contributions to the field of decision making. First, the results contribute to a better understanding of dual process models of decision making with particular emphasis on the affect heuristic. Specifically, supporting dual process theories, manipulation check results from the control condition support the notion that individuals naturally take into consideration both their feelings and the available information. Further, supporting the affect heuristic, affective impressions do appear to contribute to decision making. Current theorizing suggests that emotion-based information is stored in parallel with verbatim details and that there may be a distinct working memory subsystem for emotion (Mikels, Reuter-Lorenz, Beyer, \& Fredrickson, 2008). Thus, in the present task, it is likely that details and emotions were concurrently held in separate working memory subsystems with instructional manipulations selectively promoting one aspect over the other. Although manipulation check data provide initial support for this claim, future research is needed to better understand the relative contributions of these dual processes to decision making.

In addition, the current findings provide interesting insights into age differences in the reliance on and benefits of deliberative processes (here operationalized as a focus on facts and details). Although younger adults performed best when asked to employ information-focused strategies, results in the control condition suggest they do not naturally capitalize on such strategies under default conditions. Older adults, in contrast, reported lower reliance on facts and details across conditions and informationfocused instructions resulted in decreased choice quality. Although these patterns are only suggestive, they do shed light on interesting age differences in the dual processes involved in decision making.

From a practical point of view, the present results have important implications for the design of interventions aimed at improving decision making in older and younger adults. In advertising contexts, older adults prefer and better remember ads with emotionally meaningful appeal whereas younger adults prefer and better remember ads with knowledge-related appeal (Fung \& Carstensen, 2003). Coupled with these advertisement findings, the present results point to a need for future research that examines whether presenting health care decisions with an emphasis on information versus emotion may differentially benefit younger versus older adults. Inversely, the current results suggest that well-intended attempts to encourage older adults to focus on specific facts and details may be detrimental to their decision quality. Thus, the practical relevance of the current findings lies in the suggestion that the most effective type of decision strategy may differ for older and younger individuals.

Of course, a number of questions remain. One alternative interpretation of our findings is that participants used the same effortful/deliberative strategies across situations but that older adults' processing capacities were selectively impaired in the informationfocus condition because the instructions may have elicited stereotype threat (Hess, Auman, Colcombe, \& Rahhal, 2003; Rahhal, Hasher, \& Colcombe, 2001). Stereotype threat arises when mere awareness of a negative stereotype about one's in-group, and one's potential to reinforce it, compromises task performance by limiting processing capacity due to increased anxiety (Steele, Spencer, \& Aronson, 2002). Given that stereotype threat is associated with limitations in working memory capacity (Schmader \& Johns, 2003), this explanation requires serious consideration. Specifically, if stereotype threat were the driving factor behind our results, one would expect that fluid processing measures would be related to choice quality for older adults but not younger adults in the information-focus condition. In addition, this decrement in choice quality should be related to increased negative emotions. However, supplemental analyses revealed that in the informationfocus condition, both older and younger adults showed a significant association between choice performance and processing 
speed. Moreover, analyses of self-reported emotions indicate that older adults did not experience more negative emotions in the information-focus condition. An ANCOVA with age group as the independent variable, choice quality as the dependent variable, and negative decision-related emotions as the covariate indicated that even after accounting for negative affect, age differences in the information-focus condition remained significant, $F(1,39)=$ $19.93, p<.001, \eta_{\mathrm{p}}^{2}=.39$. Thus, although stereotype threat cannot be ruled out entirely, it cannot fully account for the current findings.

Another alternative account of the current findings is that a focus on information hurts the decision quality of older adults due to differential processing demands across conditions. In particular, it remains possible that the information-focus condition increased the processing demands of the task relative to the emotion-focus condition. Although such an interpretation could explain the decrements in decision quality for older adults, it seems less clear how such an explanation could account for the improved decision quality of younger adults in the information-focus condition. It is possible, however, that younger adults were harmed by a focus on emotion. These intriguing considerations regarding age differences in emotion-cognition interactions in decision making underscore the need for more research engendered by the current findings.

Further limitations of the current study concern the generalizability of the results and the representative nature of our decision task. Although our findings address one important domain of applied decision making (i.e., health care), it is not clear to what extent they generalize to other aspects of consumer choice. However, although the content of the present task was drawn from the specific domain of health care choices, its underlying structure was derived from a standard working memory paradigm. This design factor suggests that findings may generalize to other domains that place high load on working memory. Future research should address this important question.

In terms of representativeness, our experimental task was somewhat unusual in that it presented choice attributes in a sequential manner. This contrasts with more widely used experimental scenarios (e.g., Löckenhoff \& Carstensen, 2007) and many practical decisions in which choice attributes are presented simultaneously and can be reviewed multiple times. However, even scenarios that present information simultaneously may place high load on working memory if the amount of information is high and different options need to be compared on multiple aspects. Also, for many practical decisions, information is gathered over time and derived from multiple sources, as in the case of a prospective car buyer who might integrate information from advertisements, consumer reports, and advice from friends. Other examples for sequentially presented information are choices made over the phone or at the end of a lengthy conversation with a health care provider.

Another concern is that, beyond working memory, choices require a wide range of decision skills ranging from basic literacy and information-seeking strategies to knowing when to delegate choices that exceed one's own abilities (Löckenhoff \& Carstensen, 2004; Peters et al., 2007). For the sake of testing specific hypotheses, the present study employed a task that selectively targeted the working memory component of decision processes. Although the main effect of load suggests that we did effectively manipulate working memory, it is interesting that load did not interact with age group or instructional condition. This lack of interaction ef- fects may be partially due to a lack of power. In addition, even simplified decision tasks, such as the ones employed in the present study, may draw on additional abilities and skills that may assuage age-related decline in working memory. Future studies are needed to examine whether the effects replicate in decision tasks that target other decision making skills and processes.

In sum, the current study examined decision-making interventions aimed at eliciting deliberative versus affective processing styles and examined their influence on decision quality among younger and older individuals. The findings suggest that fairly subtle instructional manipulations were sufficient to significantly influence decision quality. Moreover, our findings provide qualified evidence that instructions encouraging affective versus deliberative processing have differential effects on the decision quality of younger and older adults. These findings imply that interventions aimed at improving decision making should be tailored to the processing abilities of the targeted age group.

\section{References}

Babcock, R. L., \& Salthouse, T. A. (1990). Effects of increased processing demands on age differences in working memory. Psychology and Aging, 5, 421-428.

Baddeley, A. D. (1986). Working memory. Oxford, England: Clarendon Press/Oxford University Press.

Baddeley, A. D., \& Hitch, G. (1974). Working memory. In G. Bower (Ed.), The psychology of learning and motivation (Vol. 8, pp. 47-89). New York, NY: Academic Press.

Baltes, P. B. (1997). On the incomplete architecture of human ontogeny: Selection, optimization, and compensation as foundation of developmental theory. American Psychologist, 52, 366-380.

Botti, S., \& Iyengar, S.-S. (2006). The dark side of choice: When choice impairs social welfare. Journal of Public Policy and Marketing, 25, 24-38.

Carstensen, L. L. (2006). The influence of a sense of time on human development. Science, 312, 1913-1915.

Carstensen, L. L., Mikels, J. A., \& Mather, M. (2006). Aging and the intersection of cognition, motivation and emotion. In J. Birren \& K. W. Schaie (Eds.), Handbook of the psychology of aging (6th ed., pp. 343-362). San Diego, CA: Academic Press.

Chasseigne, G., Mullet, E., \& Stewart, T.-R. (1997). Aging and multiple cue probability learning: The case of inverse relationships. Acta Psychologica, 97, 235-252.

Craik, F. I. M., \& Salthouse, T. A. (2008). The handbook of aging and cognition (3rd ed.). New York, NY: Psychology Press.

Daneman, M., \& Carpenter, P. A. (1980). Individual differences in working memory and reading. Journal of Verbal Learning \& Verbal Behavior, 19, 450-466.

Dror, I. E., Katona, M., \& Mungur, K. (1998). Age differences in decision making: To take a risk or not? Gerontology, 44, 67-71.

Finucane, M. L., Mertz, C. K., Slovic, P., \& Schmidt, E. S. (2005). Task complexity and older adults' decision-making competence. Psychology and Aging, 20, 71-84.

Finucane, M. L., Slovic, P., Hibbard, J. H., Peters, E., Mertz, C. K., \& MacGregor, D. G. (2002). Aging and decision-making competence: An analysis of comprehension and consistency skills in older versus younger adults considering health-plan options. Journal of Behavioral Decision Making, 15, 141-164.

Fung, H. H., \& Carstensen, L. L. (2003). Sending memorable messages to the old: Age differences in preferences and memory for advertisements. Journal of Personality and Social Psychology, 85, 163-178.

Hess, T. M., Auman, C., Colcombe, S. J., \& Rahhal, T. A. (2003). The impact of stereotype threat on age differences in memory performance. 
Journals of Gerontology: Series B: Psychological Sciences \& Social Sciences, 58B, 3-11.

Hibbard, J. H., Slovic, P., Peters, E., Finucane, M. L., \& Tusler, M. (2001). Is the informed-choice policy approach appropriate for Medicare beneficiaries? Health Affairs, 20, 199-203.

Johnson, M. M. S. (1990). Age differences in decision making: A process methodology for examining strategic information processing. Journal of Gerontology Psychological Sciences, 45, 75-78.

Johnson, M. M. S. (1993). Thinking about strategies during, before, and after making a decision. Psychology and Aging, 8, 231-241.

Kahneman, D. (2003). A perspective on judgment and choice: Mapping bounded rationality. American Psychologist, 58, 697-720.

Kennedy, Q., Mather, M., \& Carstensen, L. L. (2004). The role of motivation in the age-related positivity effect in autobiographical memory. Psychological Science, 15, 208-214.

Labouvie-Vief, G. (2003). Dynamic integration: Affect, cognition, and the self in adulthood. Current Directions in Psychological Science, 12(6), 201-206.

Löckenhoff, C. E., \& Carstensen, L. L. (2004). Socioemotional selectivity theory, aging, and health: The increasingly delicate balance between regulating emotions and making tough choices. Journal of Personality, $72,1395-1424$.

Löckenhoff, C. E., \& Carstensen, L. L. (2007). Aging, emotion, and health-related decision strategies: Motivational manipulations can reduce age differences. Psychology and Aging, 22, 134-146.

Loewenstein, G. F., Weber, E. U., Hsee, C. K., \& Welch, N. (2001). Risk as feelings. Psychological Bulletin, 127, 267-286.

Mata, R., Schooler, L. J., \& Rieskamp, J. (2007). The aging decision maker: Cognitive aging and the adaptive selection of decision strategies. Psychology and Aging, 22, 796-810.

Meyer, B. J. F., Russo, C., \& Talbot, A. (1995). Discourse comprehension and problem solving: Decisions about the treatment of breast cancer by women across the lifespan. Psychology and Aging, 9, 84-103.

Mikels, J. A., Larkin, G. R., Reuter-Lorenz, P. A., \& Carstensen, L. L. (2005). Divergent trajectories in the aging mind: Changes in working memory for affective versus visual information with age. Psychology and Aging, 20, 542-553.

Mikels, J. A., Reuter-Lorenz, P. A., Beyer, J. A., \& Fredrickson, B. L. (2008). Emotion and working memory: Evidence for domain-specific processes for affective maintenance. Emotion, 8, 256-266.

Peters, E., Hess, T. M., Vastfjall, D., \& Auman, C. (2007). Adult age differences in dual information processes: Implications for the role of affective and deliberative processes in older adults' decision making. Perspectives on Psychological Science, 2, 1-23.

Rahhal, T. A., Hasher, L., \& Colcombe, S. J. (2001). Instructional manipulations and age differences in memory: Now you see them, now you don't. Psychology and Aging, 16, 697-706.

Reyna, V. F. (2004). How people make decisions that involve risk: A dual-processes approach. Current Directions in Psychological Science, 13(2), 60-66.

Sanfey, A. G., \& Hastie, R. (2000). Judgment and decision making across the adult life span: A tutorial review of psychological research. In D. C. Park \& N. Schwarz (Eds.), Cognitive aging: A primer (pp. 253-273). New York, NY: Psychology Press.

Schmader, T., \& Johns, M. (2003). Converging evidence tat stereotype threat reduces working memory capacity. Journal of Personality and Social Psychology, 85, 440-452.

Slovic, P., Peters, E., Finucane, M. L., \& MacGregor, D. G. (2005). Affect, risk, and decision making. Health Psychology, 24, S35-S40.

Steele, C. M., Spencer, S. J., \& Aronson, J. (2002). Contending with group image: The psychology of stereotype and social identity threat. In M. P. Zanna (Ed.), Advances in experimental social psychology (Vol. 34, pp. 379-440). San Diego, CA: Academic Press.

Verhaeghen, P., Marcoen, A., \& Goossens, L. (1993). Facts and fiction about memory aging: A quantitative integration of research findings. Journals of Gerontology, 48(4), P157-P171.

Wahler, H. J. (1973). Wahler physical symptoms inventory. Los Angeles: Western Psychological Services.

Wechsler, D. (1997). Wechsler Adult Intelligence Scale (3rd ed.). San Antonio, TX: Psychological Corporation.

\section{Appendix}

Sample Trial Attributes at a Load of 8

\begin{tabular}{ll}
\hline Plan name & \multicolumn{1}{c}{ Attribute } \\
\hline Health Now & It takes little time to get reimbursed \\
Health Now & Physical therapy is not covered \\
Health Now & X-rays, CT, and MRI scans are covered \\
Health Now & It is difficult to see a specialist \\
Care Net & Vaccinations are covered \\
Care Net & No 24-hr phone hotline is available \\
Care Net & Annual physical exams are covered \\
Care Net & Quality standards for staff are high \\
\hline
\end{tabular}

Received February 23, 2009

Revision received October 20, 2009

Accepted November 12, 2009 